\title{
Umbilical cord mesenchymal stem cell transplantation in active and refractory systemic lupus erythematosus: a multicenter clinical study
}

Dandan Wang ${ }^{1}$, Jing Li ${ }^{2}$, Yu Zhang ${ }^{3}$, Miaojia Zhang ${ }^{4}$, Jinyun Chen ${ }^{1}$, Xia Lii ${ }^{1}$ Xiang $\mathrm{Hu}^{5}$, Shu Jiang ${ }^{5}$, Songtao Shi ${ }^{6}$ and Lingyun Sun ${ }^{1^{*}}$

\begin{abstract}
Introduction: In our present single-center pilot study, umbilical cord (UC)-derived mesenchymal stem cells (MSCs) had a good safety profile and therapeutic effect in severe and refractory systemic lupus erythematosus (SLE). The present multicenter clinical trial was undertaken to assess the safety and efficacy of allogeneic UC MSC transplantation (MSCT) in patients with active and refractory SLE.

Methods: Forty patients with active SLE were recruited from four clinical centers in China. Allogeneic UC MSCs were infused intravenously on days 0 and 7. The primary endpoints were safety profiles. The secondary endpoints included major clinical response (MCR), partial clinical response (PCR) and relapse. Clinical indices, including Systemic Lupus Erythematosus Disease Activity Index (SLEDAI) score, British Isles Lupus Assessment Group (BILAG) score and renal functional indices, were also taken into account.
\end{abstract}

Results: The overall survival rate was $92.5 \%$ (37 of 40 patients). UC-MSCT was well tolerated, and no transplantation-related adverse events were observed. Thirteen and eleven patients achieved MCR (13 of 40,32.5\%) and PCR (11 of 40, 27.5\%), respectively, during 12 months of follow up. Three and four patients experienced disease relapse at 9 months (12.5\%) and 12 months (16.7\%) of follow-up, respectively, after a prior clinical response. SLEDAI scores significantly decreased at 3, 6, 9 and 12 months follow-up. Total BILAG scores markedly decreased at 3 months and continued to decrease at subsequent follow-up visits. BILAG scores for renal, hematopoietic and cutaneous systems significantly improved. Among those patients with lupus nephritis, 24-hour proteinuria declined after transplantation, with statistically differences at 9 and 12 months. Serum creatinine and urea nitrogen decreased to the lowest level at 6 months, but these values slightly increased at 9 and 12 months in seven relapse cases. In addition, serum levels of albumin and complement 3 increased after MSCT, peaked at 6 months and then slightly declined by the 9 - and 12-month follow-up examinations. Serum antinuclear antibody and anti-double-stranded DNA antibody decreased after MSCT, with statistically significant differences at 3-month follow-up examinations.

Conclusion: UC-MSCT results in satisfactory clinical response in SLE patients. However, in our present study, several patients experienced disease relapse after 6 months, indicating the necessity to repeat MSCT after 6 months.

Trial registry: ClinicalTrials.gov identifier: NCT01741857. Registered 26 September 2012.

\footnotetext{
*Correspondence: lingyunsun2012@163.com

'Department of Rheumatology and Immunology, the Affiliated Drum Tower Hospital of Nanjing University Medical School, 321 Zhongshan Road, Nanjing, Jiangsu 210008, China

Full list of author information is available at the end of the article
} 


\section{Introduction}

Systemic lupus erythematosus (SLE) is a common and potentially fatal autoimmune disease characterized by autoantibodies associated with multiorgan injury, including the renal, cardiovascular, neural, musculoskeletal and cutaneous systems [1]. Although disease severity and organ involvement vary significantly among SLE patients, abnormalities of $\mathrm{T}$ and $\mathrm{B}$ lymphocytes are universal [2-4]. A deeper understanding of the underlying pathology is crucial to the development of optimal therapies for the restoration of immune homeostasis [5].

In addition to conventional immunosuppressive therapies, such as cyclophosphamide (CYC) and mycophenolate mofetil (MMF), several new strategies have been developed to target specific activation pathways relevant to SLE pathogenesis [6]. For instance, B-cell-depleting therapies using the monoclonal antibodies rituximab and the Blymphocyte stimulator (BLyS) inhibitor belimumab have been beneficial in a specific subpopulation of lupus patients [7,8]. Recently, hematopoietic stem cell transplantation (HSCT) has been reported to improve disease activity in treatment-refractory SLE [9] and in reverse organ dysfunction in several animal models [10], but the rates of relapse and treatment-related toxicity are high, as are the rates for the development of a secondary autoimmune disorder [11].

Mesenchymal stem cells (MSCs) have been widely studied as an alternative cell source for their ability to differentiate into multiple mesenchymal lineages, including bone, fat and cartilage [12]. Recent studies have indicated that these pluripotent cells can also differentiate into endoderm and neuroectoderm lineages, including neurons, hepatocytes and cardiocytes [13-15]. MSCs have been found to possess immunomodulatory effects on various activated immune cells, such as T cells, B cells, natural killer cells and dendritic cells [16-18]. Additionally, MSCs are able to escape alloantigen recognition because of their low immunogenicity and accompanying lack of expression of costimuatory molecules. These properties make MSCs promising candidate cells for preventing rejection in organ transplantation and treatment of autoimmune disease.

In recent years, we have published pilot single-center clinical studies in which we have reported the safety and efficacy of allogeneic bone marrow- or umbilical cord (UC)-derived MSCs in treating drug-resistant SLE patients, and the clinical results have been encouraging $[19,20]$. However, we had some relapsed cases during long-term follow-up [21]; thus, we found it is necessary to conduct a multicenter clinical study to further confirm the efficacy of MSC-based treatment and to explore the best effective time to initiate it in lupus patients. In our present multicenter clinical study, we found that intravenous UC MSC transplantation (MSCT) was safe and observed no transplantation-related adverse events.
UC MSC treatment resulted in clinical disease remission and systemic amelioration in lupus patients who are refractory to other. However, some patients had disease relapses after 6 months; therefore, we believe that a repeated MSC infusion is feasible and necessary after 6 months to avoid disease relapse.

\section{Methods}

\section{Patients}

From December 2009 to August 2011, 40 SLE patients ranging in age from 17 to 54 years were enrolled into our trial. Informed consent was obtained from each patient and donor. All enrolled patients met at least four of the eleven American College of Rheumatology criteria for SLE. The eligibility criteria included treatment-refractory and active disease, as well as a Systemic Lupus Erythematosus Disease Activity Index (SLEDAI) score of more than 8 or at least one British Isles Lupus Assessment Group (BILAG) grade A or at least two BILAG grade B manifestations. Refractory to treatment was defined as lack of response to treatment with monthly intravenous pulse CYC (500 to $750 \mathrm{mg} / \mathrm{m}^{2}$ ) for at least 6 months $[22,23]$, or lack of response to treatment with oral MMF $(\geq 1,000 \mathrm{mg} /$ day) [24] or leflunomide $(20 \mathrm{mg} /$ day) for at least 3 months, or continued daily doses of at least $20 \mathrm{mg}$ of prednisone (Pred) or its equivalent. Patients were excluded from the study if they had uncontrolled infection, New York Heart Association functional classification III or IV, failure of one of the vital organs or were pregnant or lactating. Active lupus nephritis (LN) was defined by meeting at least one of the following criteria: (1) laboratory tests documenting active LN three consecutive times: decrease in renal function (serum creatinine $>1.2 \mathrm{mg} / \mathrm{dl}$ ), increase in proteinuria $(>1.0 \mathrm{~g}$ of protein excretion in a 24-hour urine specimen), deterioration in microscopic hematuria ( $>10$ red blood cells per high-power field) or the presence of cellular casts; or (2) renal biopsy documenting LN according to the International Society of Nephrology/Renal Pathology Society 2003 classification system criteria for active or active/chronic LN in renal biopsy class III, class IV-S or class IV-G, class V, class III + class V or class IV + class V [25]. The study was conducted in compliance with current good clinical practice (GCP) standards and in accordance with the principles set forth under the 1989 Declaration of Helsinki. The protocol was approved by the Ethics Committee at The Drum Tower Hospital of Nanjing University Medical School, The Affiliated Hospital of Jiangsu University, Jiangsu Provincial People's Hospital and Subei People's Hospital of Jiangsu Province.

\section{Study design}

UC MSCs were prepared by the Stem Cell Center of Jiangsu Province, which is the National Stem Cell Institute in China and a member of the International Society for 
Cellular Therapy. The Stem Cell Center was also certified by the American Association of Blood Banks. Fresh UCs were obtained from informed healthy mothers in a local maternity hospital after normal deliveries. The UCs were rinsed twice in phosphate-buffered saline in penicillin and streptomycin, and the cord blood was removed during this process. The washed UCs were cut into $1-\mathrm{mm}^{2}$ pieces and floated in low-glucose Dulbecco's modified Eagle's medium containing $10 \%$ fetal bovine serum. The pieces of $\mathrm{UC}$ were subsequently incubated at $37^{\circ} \mathrm{C}$ in a humidified atmosphere consisting of $5 \% \mathrm{CO}_{2}$. Nonadherent cells were removed by washing. The medium was replaced every 3 days after the initial plating. When well-developed colonies of fibroblast-like cells appeared after about 10 days, the cells were trypsinized and passaged into a new flask for further expansion.

Cell viability was determined by trypan blue testing. The culture supernatant was analyzed for pathogenic microorganisms by direct cultivation analysis. Supernatant levels of alanine aminotransferase and endotoxins for each cell preparation were determined using an automatic biochemistry analyzer and by tachypleus amebocyte lysate analysis, respectively. In addition, supernatant virus indexes were determined by enzymelinked immunosorbent assay. Cell surface labeling markers, including CD29, CD73, CD90, CD105, CD45, CD34, CD14, CD79 and human leukocyte antigen major histocompatibility complex class II molecule, DR haplotype (HLA-DR), as well as their isotype controls, were all purchased from eBioscience (San Diego, CA, USA), and cell phenotypes were studied by flow cytometric analysis (FCM). We used good manufacturing practice conditions and clinical grade reagents to prepare the cells, and the protocol was conducted in compliance with GCP standards. One million cells per kilogram of body weight were administered by intravenous infusion on days 0 and 7.

\section{Endpoints}

Each patient returned for follow-up at 1, 3, 6, 9 and 12 months after MSCT. Evaluations performed at these follow-up visits included a physical examination, determination of SLEDAI score, BILAG analysis, serologic studies and evaluation of organ function. Adverse events and their severity were assessed and recorded throughout the study. Primary efficacy endpoints were major clinical response (MCR) and partial clinical response (PCR) assessed during the 12-month study period. A MCR was defined as achieving BILAG C scores or better in all organs at 6 months without experiencing a severe flare, which was defined, in turn, as one new domain with a BILAG A score or two new domains with BILAG B scores from MSC infusion and maintenance of this response throughout the 12-month study period. A PCR was defined as (1)
BILAG $C$ scores or better and maintenance of this response without a new BILAG A or B score within 3 months; and (2) having no more than one organ with a BILAG B score at 6 months without achieving at least one new BILAG A or B score throughout the 12-month study period [26]. No clinical response was defined as failure to meet the definition of a MCR or PCR. Clinical relapse was defined as development of at least one new domain with a BILAG A or B score after a previous MCR or PCR. Secondary efficacy endpoints included SLEDAI score, lupus serologic changes, systemic evaluations such as renal functional indexes, and hematological involvement. Transplantation-related mortality included all deaths associated with UC MSCT, except those related to recurrence of underlying disease. The investigators assessed and recorded adverse events and their severity throughout the study.

After UC MSCT, the doses of steroids as well as immunosuppressive drugs were tapered according to the amelioration of disease conditions. The dose of Pred was tapered by 5 to $10 \mathrm{mg}$ every 2 weeks during the first month following transplantation for responders. If the clinical index was not improved or if disease activity had not declined, which was defined as nonresponse, the drug dose was not tapered or new drugs might be chosen. When relapse occurred, the dose of Pred or immunosuppressive drug would be added or new drugs would be given. This protocol is uniformly adhered to at each center, and the trial was monitored by the third party (The Stem Cell Center of Jiangsu Province).

\section{Statistical analysis}

Data were analyzed as of the last data collection in August 2011. Patients were censored at the time of death or last follow-up. We used Fisher's exact test to compare the distribution of categorical variables. Pairwise comparisons of pre- and post-MSCT variables were analyzed by paired $t$-test analysis using SPSS version 13.0 statistical software (IBM SPSS, Chicago, IL, USA). The comparisons of clinical responses between patients with or without CYC treatment were analyzed by $x^{2}$ test. The BILAG index for different organ systems was used to assess response, and scores were converted to numeric values $(\mathrm{A}=9, \mathrm{~B}=3, \mathrm{C}=1, \mathrm{D}=0$ and $\mathrm{E}=0)$ to enable evaluation $[27,28]$. All $P$ values were two-sided, and $P<0.05$ was considered statistically significant.

\section{Results}

\section{Participant characteristics}

Forty patients, including thirty-eight females and two males, were enrolled in this trial. Twenty-six patients were enrolled from The Department of Rheumatology, the Affiliated Drum Tower Hospital of Nanjing University Medical School, Nanjing, China, 6, 5 and 3 patients were 
enrolled from The Department of Rheumatology, the Affiliated Hospital of Jiangsu University, Zhenjiang, China, the Department of Rheumatology, Subei People's Hospital of Jiangsu Province, Yangzhou, China, and the Department of Rheumatology, Jiangsu Provincial People's Hospital, Nanjing, China, respectively. The mean disease duration was 90.9 months, ranging from 15 to 264 months. Baseline demographics and clinical manifestations for each patient are shown in Table 1. Thirty-nine patients (39/40, 97.5\%) underwent two times of UC MSC infusions with an interval of one week, and one patient (1/40, 2.5\%) was exempted from the second MSC infusion because of uncontrolled disease progression.

\section{Umbilical cord mesenchymal stem cell characteristics}

All the infused UC MSCs were derived from passages 2 to 4 , with rigorous purification and quality control. The cell viability of purified MSCs was greater than $92 \%$. The culture supernatant was negative for pathogenic microorganisms, including aerobic and anaerobic bacteria, as well as for hepatitis B surface antigen, hepatitis B core antibody, hepatitis C virus antibody, HIV antibodies I and II, cytomegalovirus immunoglobulin $\mathrm{M}$ and syphilis antibody. FCM analysis showed CD29, CD73, CD90 and CD105 expression greater than 95\% in parallel with CD45, CD34, CD14, CD79 and HLA-DR expression less than $2 \%$. In addition, levels of alanine aminotransferase and endotoxins in the supernatants of each cell preparation were strictly controlled within $40 \mathrm{IU} / \mathrm{L}$ and 5 endotoxin units, respectively. The capacity of MSCs to differentiate into adipogenic and osteogenic lineages was also assayed.

\section{Safety}

After 12 months, the overall survival rate was $92.5 \%$ (37 of 40 patients). Three patients died as a result of uncontrolled disease activity and organ failure. One patient had active lupus with malar rash, arthralgia, uncontrolled hypertension and rapid deterioration of renal function, hypoproteinemia and severe proteinuria. She died 7 days after the first MSC infusion as a result of uncontrolled progressive disease and acute heart failure. Another patient had a lupus relapse 8 months after MSC infusion, with pulmonary hypertension, and died as a result of right-sided heart failure 256 days after MSCT. The third patient also had disease relapse 6 months after MSCT, with steroid-resistant thrombocytopenia and uncontrolled septicemia, and ultimately died due to respiratory failure 192 days after MSC infusion. Two patients had moderate herpesvirus infection 291 and 135 days after MSC treatment, respectively, and one patient had tuberculosis infection at 326 days. All the infection adverse events were treated by conventional therapies. Adverse events were not considered to be possibly related to UC MSCT. All the adverse events are listed in Table 2.

\section{Clinical outcomes \\ Clinical responses}

Thirteen and eleven patients achieved MCR (13 of 40 patients, $32.5 \%$ ) and PCR (11 of 40 patients, $27.5 \%$ ), respectively, during 12 months of follow-up. In total, 16 patients had no clinical response (16 of 40 patients, $40 \%$ ). Three and four patients, respectively, experienced disease relapse at 9 months (12.5\%) and 12 months (16.7\%) of follow-up after a prior MCR or PCR. Twentysix patients received CYC as basal treatment, and the other fourteen patients did not. However, we did not observe any difference in the rate of clinical remission between the two groups $\left(P>0.05\right.$ by $x^{2}$ test).

\section{Disease activity assessment}

Lupus disease activity, as defined by SLEDAI score, significantly decreased after MSCT (mean \pm SD values $=10.83 \pm$ 4.63 at baseline, $8.55 \pm 3.99$ at 1 month, $7.43 \pm 3.93$ at 3 months, $6.30 \pm 3.63$ at 6 months, $6.40 \pm 3.84$ at 9 months and $6.48 \pm 3.52$ at 12 months; all $P<0.01$ versus baseline levels) (Figure 1A). Total BILAG score was markedly ameliorated after UC MSC infusion (mean \pm SD values = $10.78 \pm 6.09$ at baseline, $5.35 \pm 4.48$ at 1 month, $5.28 \pm 4.71$ at 3 months, $4.23 \pm 4.43$ at 6 months, $3.85 \pm 4.73$ at 9 months and $3.55 \pm 4.33$ at 12 months; all $P<0.001$ versus baseline levels) (Figure 1B).

\section{Serology changes}

Serum albumin levels improved shortly after UC MSC infusions, were normal at the 1-month follow-up visit and remained normal during the succeeding 9 months until the 12-month follow-up visit, when they declined (mean $\pm \mathrm{SD}$ values $=3.17 \pm 0.75 \mathrm{~g} / \mathrm{dl}$ at baseline, $3.70 \pm$ $0.58 \mathrm{~g} / \mathrm{dl}$ at 1 month, $3.80 \pm 0.67 \mathrm{~g} / \mathrm{dl}$ at 3 months, $3.84 \pm$ $0.63 \mathrm{~g} / \mathrm{dl}$ at 6 months, $3.89 \pm 0.64 \mathrm{~g} / \mathrm{dl}$ at 9 months and $3.67 \pm 0.78 \mathrm{~g} / \mathrm{dl}$ at 12 months; all $P<0.05$ versus baseline levels) (Figure 2A). Serum complement 3 improved with statistical significance found at 6 months (Figure 2B). Serum complement 4 levels showed no obvious changes after MSC treatment in those patients. We observed that serum anti-double-stranded DNA antibody levels decreased after MSCT with statistically significant differences found at the 6- and 12-month follow-up visits ( mean $\pm \mathrm{SD}$ values $=710.83 \pm 814.05 \mathrm{U} / \mathrm{ml}$ at baseline, $526.78 \pm 666.7 \mathrm{U} / \mathrm{ml}$ at 1 month, $590.41 \pm 702.99 \mathrm{U} / \mathrm{ml}$ at 3 months, $492.67 \pm 615.15 \mathrm{U} / \mathrm{ml}$ at 6 months, $513.58 \pm$ $378.6 \mathrm{U} / \mathrm{ml}$ at 9 months and $212.62 \pm 244.77 \mathrm{U} / \mathrm{ml}$ at 12 months; $n=16$ ), along with decreased serum antinuclear antibody (mean \pm SD values $=5.77 \pm 2.32$ at baseline, $5.40 \pm 2.08$ at 1 month, $5.24 \pm 2.66$ at 3 months, $4.85 \pm$ 
Table 1 Clinical manifestation for each patient at baseline ${ }^{a}(n=40) *$

\begin{tabular}{|c|c|c|c|c|c|c|}
\hline $\begin{array}{l}\text { Patient/ } \\
\text { age (yr) }\end{array}$ & $\begin{array}{l}\text { Disease } \\
\text { duration (mo) }\end{array}$ & $\begin{array}{l}\text { Baseline SLEDAI } \\
\text { score }\end{array}$ & $\begin{array}{l}\text { Baseline BILAG } \\
\text { score }\end{array}$ & Total cumulative dose of IS & $\begin{array}{l}\text { Clinical outcomes } \\
\text { after MSCT }\end{array}$ & Clinical manifestations \\
\hline $1 / 46$ & 40 & 17 & 12 & CYC $0.8 \mathrm{gm} / \mathrm{mo} \times 28 \mathrm{mo}$ & PCR & LN, A, C, V, H, ANA+, anti-dsDNA+ \\
\hline $2 / 37$ & 41 & 12 & 12 & $\mathrm{CYC} 0.8 \mathrm{gm} / \mathrm{mo} \times 35 \mathrm{mo}$ & PCR & $\mathrm{A}, \mathrm{LN}, \mathrm{V}, \mathrm{ANA}+$, anti-dsDNA+, $\mathrm{H}$ \\
\hline $3 / 21$ & 50 & 11 & 9 & MMF $1.5 \mathrm{gm} / \mathrm{d} \times 31 \mathrm{mo}$ & NR & V, LN, C, anti-SM+ \\
\hline $4 / 28$ & 98 & 9 & 9 & $\begin{array}{l}\text { CYC } 0.8 \mathrm{gm} / \mathrm{mo} \times 10 \mathrm{mo}, \text { CYC } 0.8 \mathrm{gm} / \mathrm{mo} \text { combined with } \\
\text { MMF } 1.0 \mathrm{gm} / \mathrm{d} \times 28 \mathrm{mo} \text { (discontinue), LEF } 20 \mathrm{mg} / \mathrm{d} \times 31 \mathrm{mo}\end{array}$ & $M C R$ & V, A, alopecia, LN, C, ANA+, anti-dsDNA+ \\
\hline $5 / 26$ & 120 & 12 & 8 & MMF $2.0 \mathrm{gm} / \mathrm{d} \times 50 \mathrm{mo}$ (discontinue), $\mathrm{CYC} 0.8 \mathrm{gm} / \mathrm{mo} \times 20 \mathrm{mo}$ & NR & V, A, LN, ANA+, anti-dsDNA+ \\
\hline $6 / 23$ & 15 & 14 & 19 & $\mathrm{CYC} 0.8 \mathrm{gm} / \mathrm{mo} \times 15 \mathrm{mo}, \mathrm{LEF} 20 \mathrm{mg} / \mathrm{d} \times 10 \mathrm{mo}$ & NR & $V, A, F, L N, P, A N A+$, anti-dsDNA+ \\
\hline $7 / 20$ & 62 & 12 & 18 & MMF $1.5 \mathrm{gm} / \mathrm{d} \times 34 \mathrm{mo}$ (discontinued), CYC $0.8 \mathrm{gm} / \mathrm{mo} \times 24 \mathrm{mo}$ & PCR & $A, F, L N, C, P, A N A+$ \\
\hline $8 / 43$ & 26 & 34 & 20 & CYC $0.8 \mathrm{gm} / \mathrm{mo} \times 10 \mathrm{mo}$ (discontinued), LEF $20 \mathrm{mg} / \mathrm{d} \times 10 \mathrm{mo}$ & $P C R \rightarrow R$ & $C, V, L N, A$, seizures, ANA+ \\
\hline 9/36 & 97 & 10 & 26 & $\mathrm{CYC} 0.8 \mathrm{gm} / \mathrm{mo} \times 29 \mathrm{mo}$ & $M C R \rightarrow R$ & $C, V, A, L N, P, A N A+$ \\
\hline 10/39 & 60 & 10 & 7 & CYC $0.8 \mathrm{gm} / \mathrm{mo} \times 25 \mathrm{mo}$ (discontinued), LEF $20 \mathrm{mg} / \mathrm{d} \times 30 \mathrm{mo}$ & PCR & LN, A, V, ANA+, anti-SM+ \\
\hline $11 / 22$ & 40 & 8 & 16 & CYC $0.8 \mathrm{gm} / \mathrm{mo} \times 25 \mathrm{mo}$ & NR & LN, C, P, ANA+, anti-dsDNA+ \\
\hline $12 / 20$ & 50 & 14 & 13 & $\begin{array}{l}\text { CYC } 0.8 \mathrm{gm} / \mathrm{mo} \times 15 \mathrm{mo} \text { (discontinued), VCR } 1 \mathrm{mg} / \text { week } \times \\
\text { 4times (discontinued) }\end{array}$ & NR & $\begin{array}{l}\text { A, severe thrombocytopenia, V, F, ANA+, } \\
\text { anti-dsDNA+, anti-SM+ }\end{array}$ \\
\hline 13/17 & 75 & 7 & 6 & MMF $1.5 \mathrm{gm} / \mathrm{d} \times 13 \mathrm{mo}$ (discontinued), LEF $20 \mathrm{mg} / \mathrm{d} \times 30 \mathrm{mo}$ & NR & $\begin{array}{l}\text { Severe thrombocytopenia, LN, A, ANA+, } \\
\text { anti-dsDNA+ }\end{array}$ \\
\hline $14 / 21$ & 39 & 12 & 11 & CYC $0.8 \mathrm{gm} / \mathrm{mo} \times 17 \mathrm{mo}$ & NR & $L N, F, P, A$, anti-dsDNA+ \\
\hline $15 / 36$ & 60 & 10 & 7 & LEF $20 \mathrm{mg} / \mathrm{d} \times 20 \mathrm{mo}$ (discontinued), CYC $0.8 \mathrm{gm} / \mathrm{mo} \times 37 \mathrm{mo}$ & MCR & $L N, V, P, A, A N A+$, anti-SM+ \\
\hline $16 / 16$ & 49 & 11 & 15 & CYC $0.8 \mathrm{gm} / \mathrm{mo} \times 17 \mathrm{mo}$ (discontinued), LEF $20 \mathrm{mg} / \mathrm{d} \times 20 \mathrm{mo}$ & NR & $L N, A, V, A N A+$ \\
\hline $17 / 44$ & 145 & 4 & 8 & CYC $0.8 \mathrm{gm} / \mathrm{mo} \times 64 \mathrm{mo}$ & NR & $L N, A, V, C, A N A+$ \\
\hline $18 / 44$ & 85 & 8 & 9 & $\mathrm{CYC} 0.8 \mathrm{gm} / \mathrm{mo} \times 40 \mathrm{mo}$ & PCR & A, LN, F, ANAt, anti-dsDNA+ \\
\hline $19 / 29$ & 86 & 10 & 5 & CYC $0.8 \mathrm{gm} / \mathrm{mo} \times 24 \mathrm{mo}$ & $P C R \rightarrow R$ & $L N, A, P, F, A N A+$, anti-dsDNA+ \\
\hline 20/54 & 264 & 8 & 4 & $\begin{array}{l}\text { CYC } 0.8 \mathrm{gm} / \mathrm{mo} \times 36 \mathrm{mo} \text { (discontinued), MMF } 1.5 \mathrm{gm} / \mathrm{d} \times 12 \mathrm{mo} \text {, } \\
\text { then MMF } 1.0 \mathrm{gm} / \mathrm{d} \times 28 \mathrm{mo}\end{array}$ & $M C R$ & LN, A, V, C, ANA+ \\
\hline $21 / 36$ & 121 & 13 & 13 & $\mathrm{CYC} 0.8 \mathrm{gm} / \mathrm{mo} \times 25 \mathrm{mo}, \mathrm{LEF} 20 \mathrm{mg} / \mathrm{d} \times 40 \mathrm{mo}$ & $P C R \rightarrow R$ & $L N, A, V, C$ \\
\hline $22 / 40$ & 24 & 12 & 8 & CYC $0.8 \mathrm{gm} / \mathrm{mo} \times 18 \mathrm{mo}$ & NR & $F, V, L N, C, A N A+$ \\
\hline 23/35 & 25 & 14 & 24 & CYC $0.8 \mathrm{gm} / \mathrm{mo} \times 21 \mathrm{mo}$ & NR & $F, A, V, L N, P$ \\
\hline $24 / 27$ & 48 & 12 & 7 & LEF $20 \mathrm{mg} / \mathrm{d} \times 4 \mathrm{mo}$ (discontinued), CYC $0.8 \mathrm{gm} / \mathrm{mo} \times 40 \mathrm{mo}$ & MCR & $L N, F, A, P, A N A+$, anti-SM+ \\
\hline $25 / 30$ & 102 & 10 & 7 & $\begin{array}{l}\text { MMF } 2.0 \mathrm{gm} / \mathrm{d} \times 6 \mathrm{mo} \text {, then tapered to } 1.5 \mathrm{gm} / \mathrm{d} \times 36 \mathrm{mo} \text {, } \\
\text { LEF } 20 \mathrm{mg} / \mathrm{d} \times 12 \mathrm{mo} \text {, then tapered to } 10 \mathrm{mg} / \mathrm{d} \times 59 \mathrm{mo}\end{array}$ & PCR & V, A, LN, ANA+, anti-dsDNA+ \\
\hline $26 / 31$ & 62 & 8 & 3 & $\begin{array}{l}\text { MMF } 1.5 \mathrm{gm} / \mathrm{d} \times 8 \mathrm{mo} \text {, then tapered to } 1.0 \mathrm{gm} / \mathrm{d} \times 50 \mathrm{mo} \text {, } \\
\text { LEF } 20 \mathrm{mg} / \mathrm{d} \times 19 \mathrm{mo}\end{array}$ & $M C R \rightarrow R$ & $L N, V, P, A N A+$, anti-dsDNA+ \\
\hline $27 / 51$ & 108 & 13 & 29 & $\mathrm{CYC} 0.8 \mathrm{gm} / \mathrm{mo} \times 41 \mathrm{mo}, \mathrm{CsA} 150 \mathrm{mg} / \mathrm{d} \times 30 \mathrm{mo}$ & NR & $L N, V, A, C$, seizures \\
\hline $28 / 50$ & 110 & 10 & 11 & LEF 20 mg/d × $39 \mathrm{mo}$ (discontinued), CYC $0.8 \mathrm{gm} / \mathrm{mo} \times 36 \mathrm{mo}$ & MCR & $A, V, L N, A N A+$ \\
\hline
\end{tabular}


Table 1 Clinical manifestation for each patient at baseline ${ }^{a}(n=40) *($ Continued)

\begin{tabular}{|c|c|c|c|c|c|c|}
\hline 29/45 & 102 & 10 & 9 & CYC $1.2 \mathrm{gm} / \mathrm{mo} \times 22 \mathrm{mo}$ & NR & $A, V, L N, A N A+$, anti-dsDNA+ \\
\hline $30 / 33$ & 62 & 10 & 9 & CYC $0.8 \mathrm{gm} / \mathrm{mo} \times 21 \mathrm{mo}$, LEF $20 \mathrm{mg} / \mathrm{d} \times 12 \mathrm{mo}$ & MCR & $L N, A, P, C$ \\
\hline $31 / 32$ & 156 & 14 & 12 & $\begin{array}{l}\mathrm{CYC} 0.8 \mathrm{gm} / \mathrm{mo} \times 36 \mathrm{mo} \text { (discontinued), MMF } 1.5 \mathrm{gm} / \mathrm{d} \times 6 \mathrm{mo} \text {, } \\
\text { then tapered to } 1.0 \mathrm{gm} / \mathrm{d} \times 56 \mathrm{mo}\end{array}$ & $M C R \rightarrow R$ & $L N, A, C, P$ \\
\hline $32 / 53$ & 146 & 12 & 10 & $\begin{array}{l}\text { CYC } 0.8 \mathrm{gm} / \mathrm{mo} \times 24 \mathrm{mo} \text {, then tapered to } 0.6 \mathrm{gm} / \mathrm{mo} \times 42 \mathrm{mo} \text {, } \\
\text { LEF } 20 \mathrm{mg} / \mathrm{d} \times 12 \mathrm{mo} \text {, then tapered to } 10 \mathrm{mg} / \mathrm{d} \times 40 \mathrm{mo}\end{array}$ & NR & LN, A, C, anti-dsDNA+ \\
\hline $33 / 30$ & 157 & 8 & 7 & CYC $0.8 \mathrm{gm} / \mathrm{mo} \times 16 \mathrm{mo}$ (discontinued) & NR & $V, L N, A, C, H$ \\
\hline $34 / 35$ & 123 & 10 & 9 & CYC $0.8 \mathrm{gm} / \mathrm{mo} \times 18 \mathrm{mo}$, LEF $20 \mathrm{mg} / \mathrm{d} \times 22 \mathrm{mo}$ & NR & LN, A, H, C, ANA+ \\
\hline $35 / 33$ & 216 & 10 & 3 & $\mathrm{CYC} 0.8 \mathrm{gm} / \mathrm{mo} \times 26 \mathrm{mo}$ & PCR & $F, L N, V, C, A$ \\
\hline $36 / 39$ & 99 & 5 & 7 & $\mathrm{CYC} 0.8 \mathrm{gm} / \mathrm{mo} \times 14 \mathrm{mo}$ & MCR & $L N, C, V$, anti-dsDNA+ \\
\hline $37 / 35$ & 109 & 6 & 6 & LEF $20 \mathrm{mg} / \mathrm{d} \times 34 \mathrm{mo}$ & $P C R \rightarrow R$ & LN, C, H, ANA+ \\
\hline $38 / 31$ & 160 & 9 & 7 & LEF $20 \mathrm{mg} / \mathrm{d} \times 13 \mathrm{mo}$ (discontinued), CYC $0.8 \mathrm{gm} / \mathrm{mo} \times 35 \mathrm{mo}$ & MCR & $L N, C, V, A$ \\
\hline $39 / 50$ & 108 & 10 & 12 & $\mathrm{CYC} 0.8 \mathrm{gm} / \mathrm{mo} \times 14 \mathrm{mo}$ & MCR & $F, L N, A, V, C$ \\
\hline 40/35 & 96 & 8 & 8 & CYC $0.8 \mathrm{gm} / \mathrm{mo} \times 28 \mathrm{mo}$ (discontinued), LEF $20 \mathrm{mg} / \mathrm{d} \times 7 \mathrm{mo}$ & MCR & LN, V, P, C, ANA+ \\
\hline
\end{tabular}

${ }^{2}$ A, Arthralgia; ANA, Antinuclear antibody; Anti-dsDNA, anti-double-stranded DNA antibody; C, Cytopenia; F, Febrile; H, Hypocomplementemia; IS, Immunosuppressive drug; LN, Lupus nephritis; MCR, Major clinical response; NR, Nonresponse; P, Polyserositis; PCR, Partial clinical response; R, Relapse; V, Vasculitis; VCR, Vincristine. Patients' clinical manifestations were recorded within 1 week before umbilical cord mesenchymal stem cell transplantation. Total patient population $=40$. Patients 1 to 26 were enrolled from the Department of Rheumatology, Affiliated Drum Tower Hospital, Nanjing University Medical School, Nanjing, China. Patients 27 to 32 were enrolled from the Department of Rheumatology, Affiliated Hospital of Jiangsu University, Zhenjiang, China. Patients 33 to 37 were enrolled from the Department of Rheumatology, Subei People's Hospital of Jiangsu Province, Yangzhou, China. Patients 38 to 40 were enrolled from the Department of Rheumatology, Jiangsu Provincial People's Hospital, Nanjing, China. 
Table 2 Adverse events by umbilical cord mesenchymal stem cell treatment within 12 months ${ }^{a}$

\begin{tabular}{lllll}
\hline Patient & Adverse events & Severity & Time (days) & Related to MSCT \\
\hline 2 & Herpesvirus infection & AE & 291 & No relation \\
3 & Herpesvirus infection & AE & 135 & No relation \\
3 & Herpesvirus infection & AE & 187 & No relation \\
9 & Death & SAE & 7 & No relation \\
12 & Tuberculosis infection & AE & 326 & No relation \\
14 & Death & SAE & 256 & No relation \\
27 & Death & SAE & 192 & No relation \\
\hline
\end{tabular}

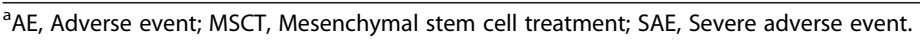

2.83 at 6 months, $4.46 \pm 2.21$ at 9 months and $4.73 \pm 2.36$ at 12 months) (Figures $2 \mathrm{C}$ and D).

\section{Organ function improvement}

Thirty-eight (95\%) of forty patients had active LN (renal BILAG A or B score) at baseline, but their renal BILAG scores decreased significantly after two UC MSC infusions (Figure 3A). Twenty-four-hour proteinuria levels decreased significantly after UC MSC treatment (mean \pm SD values $=$ $2.24 \pm 1.43 \mathrm{~g}$ at baseline, $2.13 \pm 1.35 \mathrm{~g}$ at 1 month, $1.91 \pm$ $1.20 \mathrm{~g}$ at 3 months, $1.65 \pm 1.11 \mathrm{~g}$ at 6 months, $1.24 \pm 1.09 \mathrm{~g}$ at 9 months and $1.41 \pm 1.33 \mathrm{~g}$ at 12 months; $P<0.05$ at 9- and 12-month follow-up visits) (Figure 3B). Renal function index, as assessed by serum creatinine and blood urea nitrogen levels, also decreased, and both showed statistically significant differences at the 6-month follow-up visit (Figures 3C and D), but they increased at the 12-month follow-up visit. Twenty-five (62.5\%) and twenty-eight (70\%) of the forty patients had hematopoietic and cutaneous system involvement, respectively, at baseline. The BILAG score for the two systems also ameliorated after MSC treatment (Figures 3E and F).
Therapy schedule after umbilical cord mesenchymal stem cell infusion

The dose of Pred was tapered from 5 to $10 \mathrm{mg}$ every 2 weeks during the first month following transplantation, according to clinical status and laboratory indicators of disease amelioration. During the 12-month follow-up visits, $30(81.08 \%)$ of 37 patients underwent steroid tapering, and, though 19 (54.29\%) of 35 patients underwent immunosuppressant tapering after MSCT, two patients were excluded because they had not been taking immunosuppressive drugs at baseline (Table 3).

\section{Discussion}

MSCs are multipotent, nonhematopoietic progenitor cells that are currently being explored as a promising new treatment for tissue regeneration. Although their immunomodulatory properties are not yet completely understood, their low immunogenic potential, together with their effects on immune responses, make them a promising therapeutic tool for the treatment of patients with severe and refractory autoimmune diseases. MSCs have already been applied in clinical treatment for acute graft-

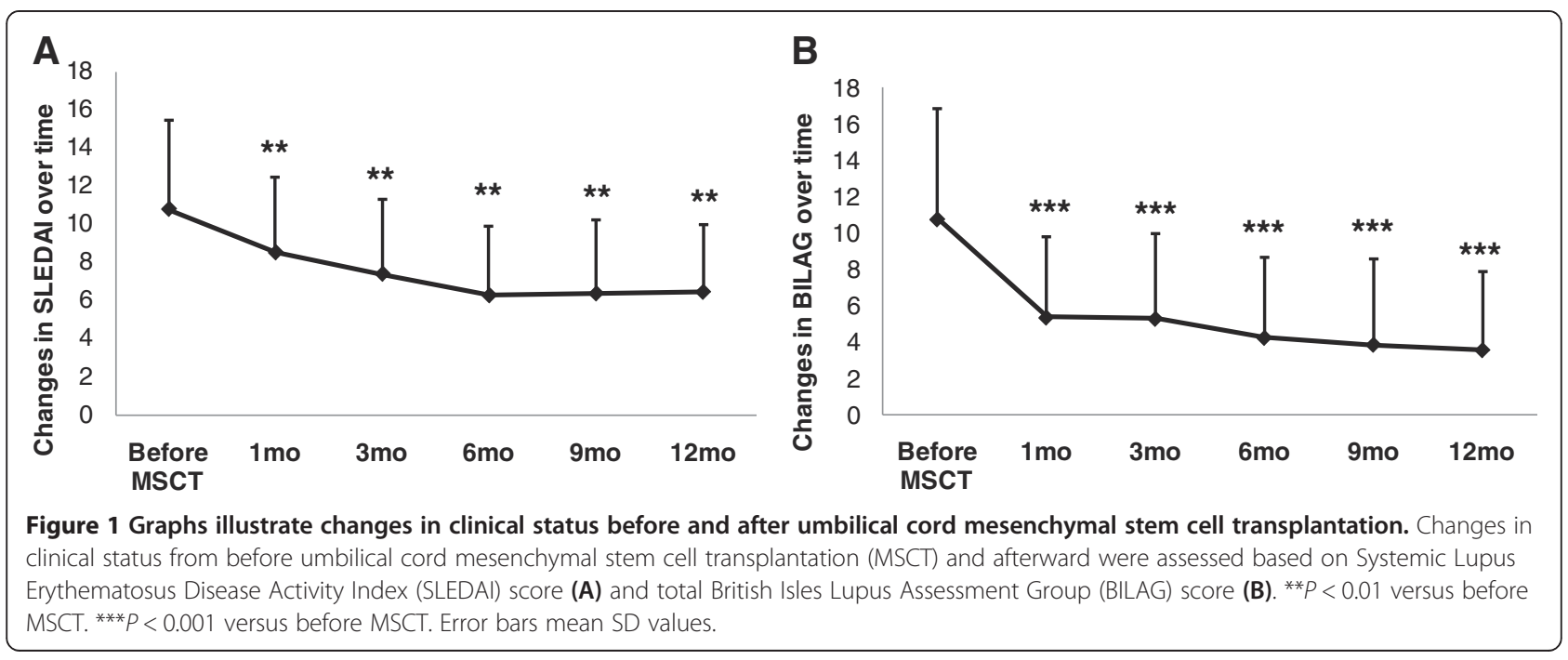




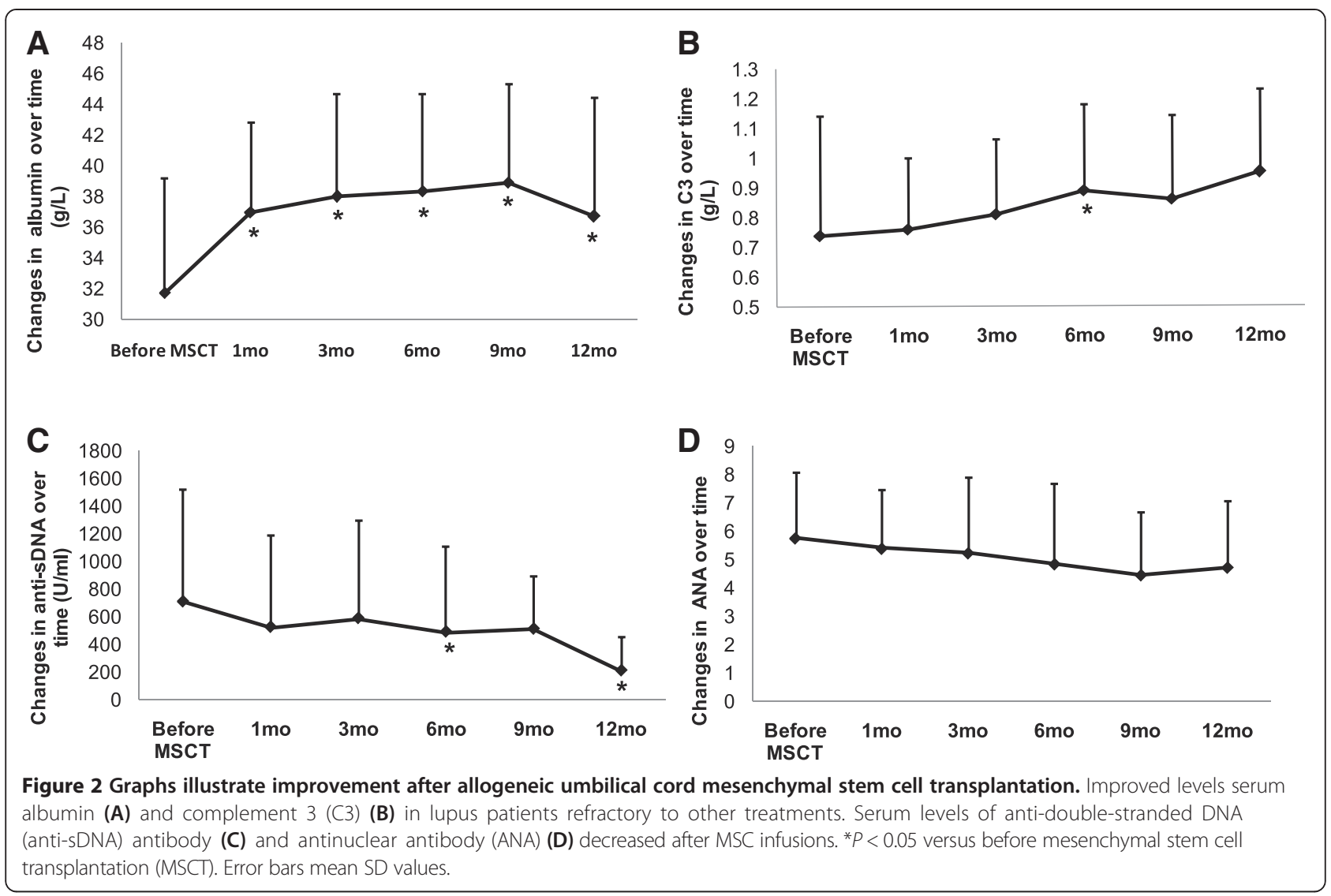

versus-host disease following allogeneic HSCT $[29,30]$, ischemic cardiomyopathy [31,32] and autoimmune diseases such as systemic sclerosis [33], inflammatory bowel disease [34,35], dermatomyositis/polymyositis [36], rheumatoid arthritis [37], Sjögren's syndrome [38] and type 1 or type 2 diabetes mellitus $[39,40]$.

To date, to the best of our knowledge, only limited clinical investigations of MSC treatment in lupus patients have been conducted. We previously conducted a smallscale, short-term study of intravenous delivery of UC MSCs [19]. Recently, a larger-scale study of 87 lupus cases and long-term follow-up of 4 years explored the clinical responses to allogeneic MSCT [21]. However, we did not have the evidence of a multicenter study to further confirm the results. Our present multicenter study has substantiated the clinical safety and efficacy of UC MSCT for the treatment of lupus patients, as determined previously in single-center studies. Sixty percent of patients achieved MCR or PCR after 12 months of follow-up, and another $40 \%$ had no clinical response. Intravenous infusion of UC MSCs is a safe practice with treatment efficacy in improving renal function and serologic indices. In addition to a significant decline of disease activity as assessed by SLEDAI and BILAG scores, UC MSC infusion ameliorated systemic manifestations in hematopoietic and cutaneous systems.
We previously compared the clinical efficacy of single and double MSC infusions in lupus patients, and the results showed that the treatment efficacy was comparable between the two groups [41]. In our present multicenter study, 39 of the 40 enrolled patients received double UC MSC infusions with a 1-week interval. At 12 months of follow-up, we found that the clinical response rate and safety profile were comparable. The results further indicate that a single infusion is enough in clinical treatment to be effective.

However, the role of MSCs in vivo is not permanent. In the present study, $12.5 \%$ and $16.7 \%$ of patients had disease relapses at 9 and 12 months of follow-up, respectively, after a prior MCR or PCR. Serologic indices, such as serum albumin and complement 3 levels, reverted slightly toward baseline, concomitantly with relapsed renal function indices, on the basis of serum creatinine and blood urea nitrogen levels. On the basis of the safety profile of MSC infusion in clinical applications, our data suggest the necessity of repeating MSC infusions after 6 months in refractory lupus patients.

MSCs can be isolated from many tissues, including bone marrow, UC, UC blood, placenta or adipose tissue. Bone marrow-derived MSCs, both autologous and allogeneic, are widely used in clinical applications. However, an increasing number of recent studies have shown that 


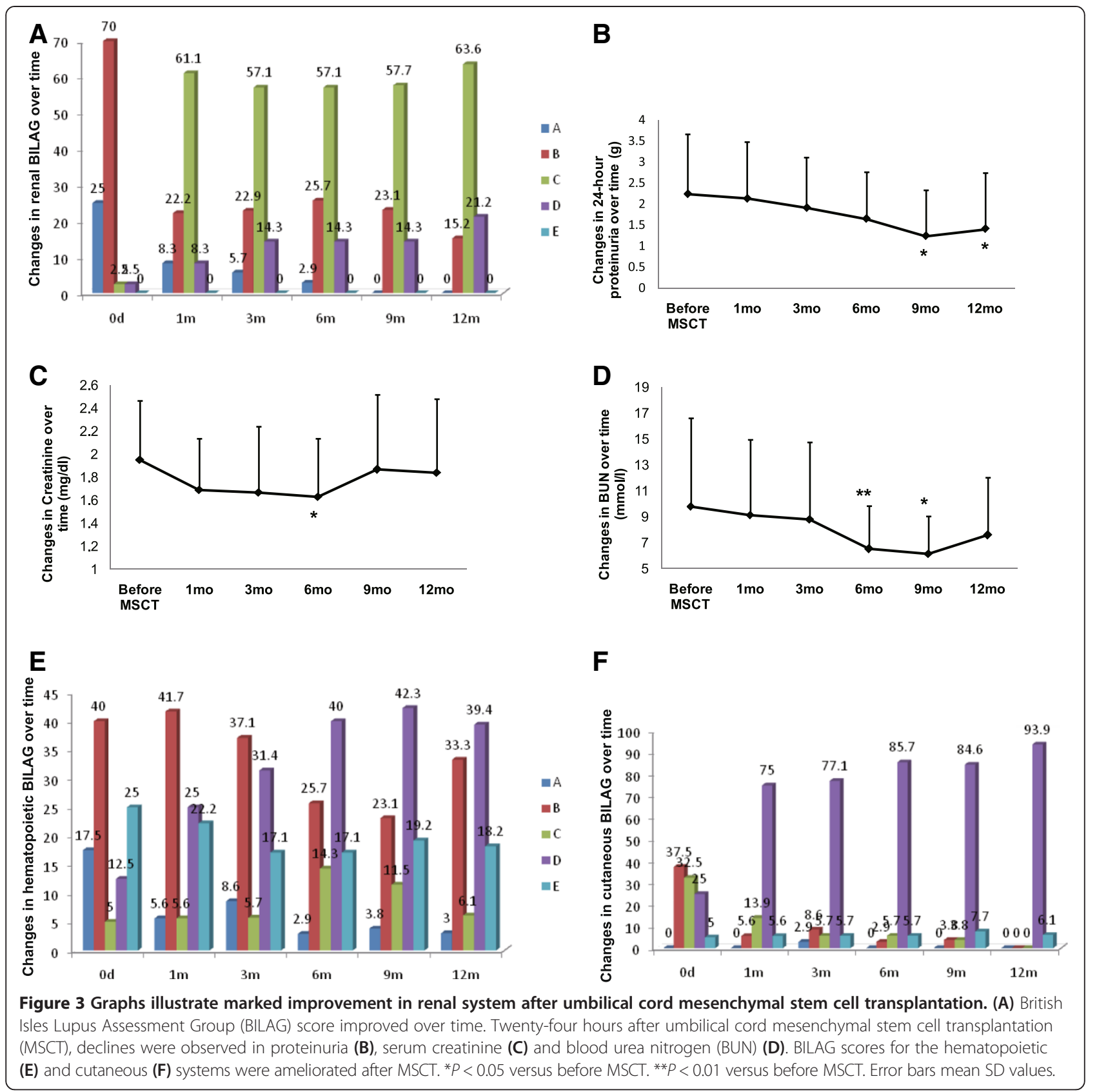

MSCs from bone marrow are difficult to obtain, have ethical issues and are easily contaminated. Moreover, autologous bone marrow-derived MSCs are functionally abnormal in some disorders such as lupus [42,43], rheumatoid arthritis [44] and systemic sclerosis [45], which may limit their clinical application. UCs fall off after delivery, but they are rich in MSCs. UC MSCs have many advantages over bone marrow MSCs, including easy access, less possibility of contamination and no ethical problems. Furthermore, UC MSCs, in contrast to bone marrow MSCs, do not express tumor-associated fibroblast phenotypes and therefore have no opportunity to grow solid tumors [46]. Moreover, UC MSCs have a higher rate of gene expression related to cell adhesion, morphogenesis, angiogenesis and neurogenesis than UC blood-derived MSCs do [47], and they can accumulate more mineralized matrix than placenta-derived MSCs [48], indicating that UC MSCs may be used as an optimal cell therapy option.

The present study has some limitations. First, 95\% of the patients had active LN at the time of study entry, but we cannot provide the pathologic data of the present enrolled patients. Therefore, we do not know whether MSCs can indeed ameliorate renal pathology, aside from 
Table 3 Treatments used before and after umbilical cord mesenchymal stem cell transplantation in each patient ${ }^{\mathrm{a}}$

\begin{tabular}{|c|c|c|c|c|c|}
\hline Patient & Baseline & 1 month & 3 months & 6 months & 12 months \\
\hline \multirow[t]{4}{*}{1} & Pred $5 \mathrm{mg} / \mathrm{d}$ & Pred 5 mg/d, & Pred $5 \mathrm{mg} / \mathrm{d}$ & Pred $5 \mathrm{mg} / \mathrm{d}$ & Pred $5 \mathrm{mg} / \mathrm{d}$ \\
\hline & CYC $0.8 \mathrm{~g} / \mathrm{mo}$ & CYC 0.8 g/mo, & CYC $0.8 \mathrm{~g} / \mathrm{mo}$ & CYC $0.8 \mathrm{~g} / \mathrm{mo}$ & CYC $0.8 \mathrm{~g} / \mathrm{mo}$ \\
\hline & LEF 20 mg/d & LEF 20 mg/d, & LEF $20 \mathrm{mg} / \mathrm{d}$ & LEF $20 \mathrm{mg} / \mathrm{d}$ & LEF 20 mg/d \\
\hline & HCQ 200 mg/d & HCQ 200 mg/d & HCQ 200 mg/d & HCQ 200 mg/d & HCQ 200 mg/d \\
\hline \multirow[t]{2}{*}{2} & Pred $30 \mathrm{mg} / \mathrm{d}$ & Pred 15 mg/d & Pred 10 mg/d & Pred $10 \mathrm{mg} / \mathrm{d}$ & Pred $10 \mathrm{mg} / \mathrm{d}$ \\
\hline & $\mathrm{CYC} 0.8 \mathrm{~g} / \mathrm{mo}$ & CYC $0.8 \mathrm{~g} / \mathrm{mo}$ & CYC $0.8 \mathrm{~g} / \mathrm{mo}$ & CYC $0.8 \mathrm{~g} / \mathrm{mo}$ & CYC $0.8 \mathrm{~g} / \mathrm{mo}$ \\
\hline \multirow[t]{3}{*}{3} & Pred 15 mg/d & Pred 15 mg/d & Pred 10 mg/d & Pred 15 mg/d & Pred 15 mg/d \\
\hline & MMF $1.5 \mathrm{~g} / \mathrm{d}$ & MMF $1.5 \mathrm{~g} / \mathrm{d}$ & MMF $1.5 \mathrm{~g} / \mathrm{d}$ & MMF $1.5 \mathrm{~g} / \mathrm{d}$ & MMF $1.0 \mathrm{~g} / \mathrm{d}$ \\
\hline & HCQ 200 mg/d & HCQ 200 mg/d & HCQ 200 mg/d & HCQ 200 mg/d & HCQ 200 mg/d \\
\hline \multirow[t]{3}{*}{4} & Pred $10 \mathrm{mg} / \mathrm{d}$ & Pred $5 \mathrm{mg} / \mathrm{d}$ & Pred $5 \mathrm{mg} / \mathrm{d}$ & Pred $5 \mathrm{mg} / \mathrm{d}$ & Pred $5 \mathrm{mg} / \mathrm{d}$ \\
\hline & LEF 20 mg/d & LEF 20 mg/d & LEF 20 mg/d & LEF $10 \mathrm{mg} / \mathrm{d}$ & LEF $10 \mathrm{mg} / \mathrm{d}$ \\
\hline & HCQ 400 mg/d & HCQ 400 mg/d & HCQ 400 mg/d & HCQ 400 mg/d & HCQ 200 mg/d \\
\hline \multirow[t]{3}{*}{5} & Pred $10 \mathrm{mg} / \mathrm{d}$ & Pred 10 mg/d & Pred 5 mg/d & Pred 5 mg/d & Pred 5 mg/d \\
\hline & $\mathrm{CYC} 0.8 \mathrm{~g} / \mathrm{mo}$ & CYC $0.8 \mathrm{~g} / \mathrm{mo}$ & CYC 0.8 g/mo & CYC $0.8 \mathrm{~g} / \mathrm{mo}$ & CYC $0.6 \mathrm{~g} / \mathrm{mo}$ \\
\hline & $\mathrm{HCQ} 400 \mathrm{mg} / \mathrm{d}$ & HCQ 400 mg/d & $\mathrm{HCQ} 400 \mathrm{mg} / \mathrm{d}$ & HCQ 400 mg/d & HCQ 400 mg/d \\
\hline \multirow[t]{5}{*}{6} & Pred 20 mg/d & Pred 20 mg/d & Pred 15 mg/d & Pred 15 mg/d & Pred 15 mg/d \\
\hline & $\mathrm{CYC} 0.8 \mathrm{~g} / \mathrm{mo}$ & CYC $0.8 \mathrm{~g} / \mathrm{mo}$ & CYC 0.8 g/mo & CYC $0.8 \mathrm{~g} / \mathrm{mo}$ & CYC $0.8 \mathrm{~g} / \mathrm{mo}$ \\
\hline & LEF 20 mg/d & LEF 20 mg/d & LEF 20 mg/d & LEF 20 mg/d & LEF 20 mg/d \\
\hline & $\mathrm{HCQ} 400$ mg/d & HCQ 400 mg/d & $\mathrm{HCQ} 400 \mathrm{mg} / \mathrm{d}$ & HCQ 400 mg/d & HCQ 400 mg/d \\
\hline & & & & Triptolide 60 mg/d & Triptolide 60 mg/d \\
\hline \multirow[t]{3}{*}{7} & Pred 15 mg/d & Pred 10 mg/d & Pred 10 mg/d & Pred $10 \mathrm{mg} / \mathrm{d}$ & Pred $10 \mathrm{mg} / \mathrm{d}$ \\
\hline & $\mathrm{CYC} 0.8 \mathrm{~g} / \mathrm{mo}$ & $\mathrm{CYC} 0.8 \mathrm{~g} / \mathrm{mo}$ & CYC 0.8 g/mo & $\mathrm{CYC} 0.8 \mathrm{~g} / \mathrm{mo}$ & CYC $0.6 \mathrm{~g} / \mathrm{mo}$ \\
\hline & HCQ 300 mg/d & HCQ 300 mg/d & HCQ 300 mg/d & HCQ 300 mg/d & HCQ 300 mg/d \\
\hline \multirow[t]{3}{*}{8} & Pred $20 \mathrm{mg} / \mathrm{d}$ & Pred 15 mg/d & Pred 15 mg/d & Pred 30 mg/d & Pred $10 \mathrm{mg} / \mathrm{d}$ \\
\hline & LEF 20 mg/d & LEF 20 mg/d & LEF 20 mg/d & LEF 20 mg/d & LEF 20 mg/d \\
\hline & HCQ 400 mg/d & HCQ 400 mg/d & HCQ 400 mg/d & HCQ 400 mg/d & HCQ 300 mg/d \\
\hline \multirow[t]{3}{*}{9} & Pred 40 mg/d & Pred 25 mg/d & Pred 15 mg/d & Pred $10 \mathrm{mg} / \mathrm{d}$ & / \\
\hline & CYC $0.8 \mathrm{~g} / \mathrm{mo}$ & CYC $0.8 \mathrm{~g} / \mathrm{mo}$ & CYC $0.8 \mathrm{~g} / \mathrm{mo}$ & CYC $0.8 \mathrm{~g} / \mathrm{mo}$ & \\
\hline & HCQ 400 mg/d & HCQ 400 mg/d & HCQ 400 mg/d & HCQ 400 mg/d & \\
\hline \multirow[t]{3}{*}{10} & Pred $10 \mathrm{mg} / \mathrm{d}$ & Pred $5 \mathrm{mg} / \mathrm{d}$ & Pred 5 mg/d & Pred 5 mg/d & Pred 5 mg/d \\
\hline & LEF 20 mg/d & LEF 20 mg/d & LEF $10 \mathrm{mg} / \mathrm{d}$ & LEF 10 mg/d & LEF 10 mg/d \\
\hline & HCQ 400 mg/d & HCQ 400 mg/d & HCQ 400 mg/d & HCQ 400 mg/d & HCQ 400 mg/d \\
\hline \multirow[t]{3}{*}{11} & Pred $20 \mathrm{mg} / \mathrm{d}$ & Pred 20 mg/d & Pred 15 mg/d & Pred $10 \mathrm{mg} / \mathrm{d}$ & Pred $10 \mathrm{mg} / \mathrm{d}$ \\
\hline & CYC 0.8 g/mo & $\mathrm{CYC} 0.8 \mathrm{~g} / \mathrm{mo}$ & CYC 0.8 g/mo & CYC 0.8 g/mo & CYC $0.4 \mathrm{~g} / \mathrm{mo}$ \\
\hline & & HCQ 400 mg/d & HCQ 400 mg/d & HCQ 400 mg/d & HCQ 400 mg/d \\
\hline \multirow[t]{2}{*}{12} & Pred $20 \mathrm{mg} / \mathrm{d}$ & Pred 15 mg/d & Pred 15 mg/d & Pred $10 \mathrm{mg} / \mathrm{d}$ & Pred $10 \mathrm{mg} / \mathrm{d}$ \\
\hline & HCQ 400 mg/d & HCQ 400 mg/d & HCQ 400 mg/d & HCQ 400 mg/d & HCQ 400 mg/d \\
\hline \multirow[t]{3}{*}{13} & Pred 20 mg/d & Pred $15 \mathrm{mg} / \mathrm{d}$ & Pred 15 mg/d & Pred $10 \mathrm{mg} / \mathrm{d}$ & Pred $10 \mathrm{mg} / \mathrm{d}$ \\
\hline & LEF 20 mg/d & LEF 20 mg/d & LEF 20 mg/d & LEF 20 mg/d & LEF 20 mg/d \\
\hline & $\mathrm{HCQ} 400 \mathrm{mg} / \mathrm{d}$ & $\mathrm{HCQ} 400 \mathrm{mg} / \mathrm{d}$ & $\mathrm{HCQ} 400 \mathrm{mg} / \mathrm{d}$ & HCQ 400 mg/d & HCQ 400 mg/d \\
\hline \multirow[t]{2}{*}{14} & Pred $15 \mathrm{mg} / \mathrm{d}$ & / & / & / & / \\
\hline & CYC $0.8 \mathrm{~g} / \mathrm{mo}$ & & & & \\
\hline
\end{tabular}


Table 3 Treatments used before and after umbilical cord mesenchymal stem cell transplantation in each patient ${ }^{a}$ (Continued)

\begin{tabular}{|c|c|c|c|c|c|}
\hline \multirow[t]{3}{*}{15} & Pred 15 mg/d & Pred 15 mg/d & Pred 15 mg/d & Pred $10 \mathrm{mg} / \mathrm{d}$ & Pred $10 \mathrm{mg} / \mathrm{d}$ \\
\hline & CYC $0.8 \mathrm{~g} / \mathrm{mo}$ & CYC $0.8 \mathrm{~g} / \mathrm{mo}$ & CYC 0.8 g/mo, & CYC0.8 g/mo, & CYC $0.4 \mathrm{~g} / \mathrm{mo}$ \\
\hline & HCQ 400 mg/d & HCQ 400 mg/d & HCQ 300 mg/d & HCQ 300 mg/d & HCQ 300 mg/d \\
\hline \multirow[t]{3}{*}{16} & Pred 15 mg/d & Pred 15 mg/d & Pred 10 mg/d & Pred $10 \mathrm{mg} / \mathrm{d}$ & Pred 20 mg/d \\
\hline & LEF 20 mg/d & LEF 20 mg/d & LEF 20 mg/d & LEF 20 mg/d & LEF 20 mg/d \\
\hline & HCQ 200 mg/d & HCQ 200 mg/d & HCQ 200 mg/d & HCQ 200 mg/d & HCQ $200 \mathrm{mg} / \mathrm{d}$ \\
\hline \multirow[t]{3}{*}{17} & Pred 15 mg/d & Pred 15 mg/d & Pred 15 mg/d & Pred $10 \mathrm{mg} / \mathrm{d}$ & Pred $10 \mathrm{mg} / \mathrm{d}$ \\
\hline & CYC $0.8 \mathrm{~g} / \mathrm{mo}$ & CYC $0.8 \mathrm{~g} / \mathrm{mo}$ & CYC $0.8 \mathrm{~g} / 45 \mathrm{~d}$ & CYC $0.8 \mathrm{~g} / 45 \mathrm{~d}$ & CYC $0.4 \mathrm{~g} / \mathrm{mo}$ \\
\hline & HCQ 200 mg/d & HCQ 200 mg/d & HCQ $200 \mathrm{mg} / \mathrm{d}$ & HCQ 200 mg/d & HCQ 200 mg/d \\
\hline \multirow[t]{3}{*}{18} & Pred 20 mg/d & Pred 15 mg/d & Pred 15 mg/d & Pred 15 mg/d & Pred $10 \mathrm{mg} / \mathrm{d}$ \\
\hline & CYC $0.8 \mathrm{~g} / \mathrm{mo}$ & CYC $0.8 \mathrm{~g} / \mathrm{mo}$ & CYC $0.8 \mathrm{~g} / \mathrm{mo}$ & CYC $0.8 \mathrm{~g} / \mathrm{mo}$ & CYC $0.8 \mathrm{~g} / \mathrm{mo}$ \\
\hline & $\mathrm{HCQ} 400 \mathrm{mg} / \mathrm{d}$ & HCQ 400 mg/d & HCQ $400 \mathrm{mg} / \mathrm{d}$ & HCQ 400 mg/d & HCQ $400 \mathrm{mg} / \mathrm{d}$ \\
\hline \multirow[t]{3}{*}{19} & Pred 15 mg/d & Pred 15 mg/d & Pred 10 mg/d & Pred $10 \mathrm{mg} / \mathrm{d}$ & Pred 15 mg/d \\
\hline & CYC 0.8 g/mo & CYC $0.8 \mathrm{~g} / \mathrm{mo}$ & CYC 0.8 g/mo & CYC $0.6 \mathrm{~g} / \mathrm{mo}$ & CYC $0.8 \mathrm{~g} / \mathrm{mo}$ \\
\hline & HCQ 400 mg/d & HCQ 400 mg/d & HCQ $400 \mathrm{mg} / \mathrm{d}$ & HCQ 400 mg/d & HCQ $400 \mathrm{mg} / \mathrm{d}$ \\
\hline \multirow[t]{3}{*}{20} & Pred $5 \mathrm{mg} / \mathrm{d}$ & Pred $5 \mathrm{mg} / \mathrm{d}$ & Pred $5 \mathrm{mg} / \mathrm{d}$ & Pred 5 mg/d & Pred 5 mg/d \\
\hline & MMF $1.0 \mathrm{~g} / \mathrm{d}$ & MMF $1.0 \mathrm{~g} / \mathrm{d}$ & MMF $1.0 \mathrm{~g} / \mathrm{d}$ & MMF $1.0 \mathrm{~g} / \mathrm{d}$ & MMF $0.5 \mathrm{~g} / \mathrm{d}$ \\
\hline & HCQ 200 mg/d & HCQ 200 mg/d & HCQ 200 mg/d & HCQ 200 mg/d & HCQ 200 mg/d \\
\hline \multirow[t]{4}{*}{21} & Pred $7.5 \mathrm{mg} / \mathrm{d}$ & Pred 7.5 mg/d & Pred 5 mg/d & Pred 15 mg/d & Pred 15 mg/d \\
\hline & CYC 0.8 g/mo & CYC $0.8 \mathrm{~g} / \mathrm{mo}$ & CYC 0.8 g/mo & CYC $0.8 \mathrm{~g} / \mathrm{mo}$ & CYC $0.8 \mathrm{~g} / \mathrm{mo}$ \\
\hline & LEF 10 mg/d & LEF 10 mg/d & LEF 10 mg/d & LEF 10 mg/d & LEF 10 mg/d \\
\hline & $\mathrm{HCQ} 400 \mathrm{mg} / \mathrm{d}$ & HCQ 400 mg/d & $\mathrm{HCQ} 400 \mathrm{mg} / \mathrm{d}$ & HCQ 400 mg/d & HCQ 200 mg/d \\
\hline 22 & $\begin{array}{l}\text { Pred } 20 \mathrm{mg} / \mathrm{d} \\
\text { CYC } 0.8 \mathrm{~g} / \mathrm{mo} \\
\text { HCQ } 400 \mathrm{mg} / \mathrm{d}\end{array}$ & $\begin{array}{l}\text { Pred } 15 \mathrm{mg} / \mathrm{d} \\
\text { CYC } 0.8 \mathrm{~g} / \mathrm{mo} \\
\text { HCQ } 400 \mathrm{mg} / \mathrm{d}\end{array}$ & $\begin{array}{l}\text { Pred } 15 \mathrm{mg} / \mathrm{d} \\
\text { CYC } 0.8 \mathrm{~g} / \mathrm{mo} \\
\text { HCQ } 400 \mathrm{mg} / \mathrm{d}\end{array}$ & $\begin{array}{l}\text { Pred } 10 \mathrm{mg} / \mathrm{d} \\
\text { CYC } 0.8 \mathrm{~g} / \mathrm{mo} \\
\text { HCQ } 400 \mathrm{mg} / \mathrm{d}\end{array}$ & $\begin{array}{l}\text { Pred } 10 \mathrm{mg} / \mathrm{d} \\
\text { CYC } 0.6 \mathrm{~g} / \mathrm{mo} \\
\text { HCQ } 400 \mathrm{mg} / \mathrm{d}\end{array}$ \\
\hline \multirow[t]{3}{*}{23} & Pred 45 mg/d & Pred $15 \mathrm{mg} / \mathrm{d}$ & Pred 10 mg/d & Pred $10 \mathrm{mg} / \mathrm{d}$ & Pred $10 \mathrm{mg} / \mathrm{d}$ \\
\hline & CYC 0.8 g/mo & $\mathrm{CYC} 0.8 \mathrm{~g} / \mathrm{mo}$ & CYC 0.8 g/mo & CYC $0.8 \mathrm{~g} / \mathrm{mo}$ & CYC $0.6 \mathrm{~g} / \mathrm{mo}$ \\
\hline & HCQ 400 mg/d & HCQ 400 mg/d & HCQ 400 mg/d & HCQ 400 mg/d & HCQ 200 mg/d \\
\hline \multirow[t]{2}{*}{24} & Pred 15 mg/d & Pred 15 mg/d & Pred 10 mg/d & Pred 5 mg/d & Pred 5 mg/d \\
\hline & CYC 0.8 g/mo & CYC $0.8 \mathrm{~g} / \mathrm{mo}$ & CYC 0.8 g/mo & CYC $0.8 \mathrm{~g} / \mathrm{mo}$ & CYC $0.6 \mathrm{~g} / \mathrm{mo}$ \\
\hline \multirow[t]{4}{*}{25} & Pred 15 mg/d & Pred $10 \mathrm{mg} / \mathrm{d}$ & Pred 10 mg/d & Pred $10 \mathrm{mg} / \mathrm{d}$ & Pred $10 \mathrm{mg} / \mathrm{d}$ \\
\hline & MMF $1.5 \mathrm{~g} / \mathrm{d}$ & MMF $1.5 \mathrm{~g} / \mathrm{d}$ & MMF $1.5 \mathrm{~g} / \mathrm{d}$ & MMF $1.0 \mathrm{~g} / \mathrm{d}$ & MMF $1.0 \mathrm{~g} / \mathrm{d}$ \\
\hline & LEF 10 mg/d & LEF 10 mg/d & $\mathrm{HCQ} 400 \mathrm{mg} / \mathrm{d}$ & HCQ 400 mg/d & HCQ $400 \mathrm{mg} / \mathrm{d}$ \\
\hline & HCQ 400 mg/d & HCQ 400 mg/d & & & \\
\hline \multirow[t]{4}{*}{26} & Pred 20 mg/d & Pred 15 mg/d & Pred 10 mg/d & Pred $10 \mathrm{mg} / \mathrm{d}$ & Pred $20 \mathrm{mg} / \mathrm{d}$ \\
\hline & MMF $1.0 \mathrm{~g} / \mathrm{d}$ & MMF $1.0 \mathrm{~g} / \mathrm{d}$ & MMF $1.0 \mathrm{~g} / \mathrm{d}$ & MMF $1.0 \mathrm{~g} / \mathrm{d}$ & MMF $1.0 \mathrm{~g} / \mathrm{d}$ \\
\hline & LEF $20 \mathrm{mg} / \mathrm{d}$ & LEF $20 \mathrm{mg} / \mathrm{d}$ & LEF $20 \mathrm{mg} / \mathrm{d}$ & LEF $20 \mathrm{mg} / \mathrm{d}$ & LEF 20 mg/d \\
\hline & HCQ 200 mg/d & HCQ 200 mg/d & HCQ 200 mg/d & HCQ 200 mg/d & HCQ $200 \mathrm{mg} / \mathrm{d}$ \\
\hline \multirow[t]{3}{*}{27} & Pred 50 mg/d & Pred $30 \mathrm{mg} / \mathrm{d}$ & Pred 15 mg/d & MP 80 mg/d & / \\
\hline & CYC $0.8 \mathrm{~g} / \mathrm{mo}$ & CYC $0.8 \mathrm{~g} / \mathrm{mo}$ & CYC 0.8 g/mo & $C Y C 0.6 \mathrm{~g} / \mathrm{d} \times 3 \mathrm{~d}$ & \\
\hline & CsA 150 mg/d & CsA 150 mg/d & CsA 150 mg/d & Y-globulin $20 \mathrm{~g} \times 3 \mathrm{~d}$ & \\
\hline \multirow[t]{3}{*}{28} & Pred 20 mg/d & Pred $15 \mathrm{mg} / \mathrm{d}$ & Pred 15 mg/d & Pred 5 mg/d & Pred 5 mg/d \\
\hline & CYC $0.8 \mathrm{~g} / \mathrm{mo}$ & CYC $0.8 \mathrm{~g} / \mathrm{mo}$ & $\mathrm{CYC} 0.8 \mathrm{~g} / \mathrm{mo}$ & CYC $0.8 \mathrm{~g} / \mathrm{mo}$ & CYC $0.8 \mathrm{~g} / \mathrm{mo}$ \\
\hline & HCQ 200 mg/d & HCQ 200 mg/d & HCQ 200 mg/d & HCQ 200 mg/d & HCQ 200 mg/d \\
\hline
\end{tabular}


Table 3 Treatments used before and after umbilical cord mesenchymal stem cell transplantation in each patient ${ }^{\mathrm{a}}$ (Continued)

\begin{tabular}{|c|c|c|c|c|c|}
\hline \multirow[t]{3}{*}{29} & Pred 30 mg/d & Pred 25 mg/d & Pred 20 mg/d & Pred $10 \mathrm{mg} / \mathrm{d}$ & Pred $5 \mathrm{mg} / \mathrm{d}$ \\
\hline & CYC 1.2 g/mo & CYC 1.2 g/mo & CYC $1.2 \mathrm{~g} / \mathrm{mo}$ & CYC $1.2 \mathrm{~g} / \mathrm{mo}$ & $\mathrm{CYC} 0.8 \mathrm{~g} / \mathrm{mo}$ \\
\hline & & & & Triptolide 60 mg/d & Triptolide 60 mg/d \\
\hline \multirow[t]{3}{*}{30} & Pred 25 mg/d & Pred 20 mg/d & Pred 15 mg/d & Pred $10 \mathrm{mg} / \mathrm{d}$ & Pred $10 \mathrm{mg} / \mathrm{d}$ \\
\hline & CYC 0.8 g/mo & CYC 0.8 g/mo & CYC0.8 g/mo & CYC0.8 g/mo & CYC0.6 g/mo \\
\hline & LEF 20 mg/d & LEF 20 mg/d & LEF 20 mg/d & LEF 20 mg/d & LEF $10 \mathrm{mg} / \mathrm{d}$ \\
\hline \multirow[t]{2}{*}{31} & Pred 30 mg/d & Pred 25 mg/d & Pred 25 mg/d & Pred $30 \mathrm{mg} / \mathrm{d}$ & Pred $15 \mathrm{mg} / \mathrm{d}$ \\
\hline & MMF $1.0 \mathrm{~g} / \mathrm{d}$ & MMF $1.0 \mathrm{~g} / \mathrm{d}$ & MMF $1.0 \mathrm{~g} / \mathrm{d}$ & MMF $1.0 \mathrm{~g} / \mathrm{d}$ & MMF $1.0 \mathrm{~g} / \mathrm{d}$ \\
\hline \multirow[t]{4}{*}{32} & Pred 25 mg/d & Pred 20 mg/d & Pred 15 mg/d & Pred 15 mg/d & Pred 15 mg/d \\
\hline & CYC 0.6 g/mo & CYC 0.6 g/mo & CYC 0.6 g/mo & CYC $0.6 \mathrm{~g} / \mathrm{mo}$ & CYC $0.6 \mathrm{~g} / \mathrm{mo}$ \\
\hline & LEF $10 \mathrm{mg} / \mathrm{d}$ & LEF 10 mg/d & LEF 10 mg/d & LEF $10 \mathrm{mg} / \mathrm{d}$ & LEF $10 \mathrm{mg} / \mathrm{d}$ \\
\hline & HCQ 400 mg/d & HCQ 400 mg/d & $\mathrm{HCQ} 400 \mathrm{mg} / \mathrm{d}$ & $\mathrm{HCQ} 400 \mathrm{mg} / \mathrm{d}$ & HCQ 200 mg/d \\
\hline 33 & Pred 25 mg/d & Pred 15 mg/d & Pred 15 mg/d & Pred 35 mg/d & Pred 35 mg/d \\
\hline \multirow[t]{3}{*}{34} & Pred 15 mg/d & Pred 15 mg/d & Pred 15 mg/d & Pred $15 \mathrm{mg} / \mathrm{d}$ & Pred $10 \mathrm{mg} / \mathrm{d}$ \\
\hline & CYC $0.8 \mathrm{~g} / \mathrm{mo}$ & CYC 0.8 g/mo & CYC 0.8 g/mo & CYC $0.8 \mathrm{~g} / \mathrm{mo}$ & CYC $0.8 \mathrm{~g} / \mathrm{mo}$ \\
\hline & LEF 20 mg/d & LEF 20 mg/d & LEF 10 mg/d & LEF 10 mg/d & LEF $10 \mathrm{mg} / \mathrm{d}$ \\
\hline \multirow[t]{2}{*}{35} & Pred 20 mg/d, & Pred 15 mg/d & Pred 15 mg/d & Pred $10 \mathrm{mg} / \mathrm{d}$ & Pred $10 \mathrm{mg} / \mathrm{d}$ \\
\hline & CYC $0.8 \mathrm{~g} / \mathrm{mo}$ & CYC $0.8 \mathrm{~g} / \mathrm{mo}$ & CYC 0.8 g/mo & CYC $0.8 \mathrm{~g} / \mathrm{mo}$ & CYC $0.6 \mathrm{~g} / \mathrm{mo}$ \\
\hline \multirow[t]{3}{*}{36} & Pred 25 mg/d & Pred 15 mg/d & Pred 15 mg/d & Pred $10 \mathrm{mg} / \mathrm{d}$ & Pred $10 \mathrm{mg} / \mathrm{d}$ \\
\hline & CYC 0.8 g/mo & CYC 0.8 g/mo & CYC 0.8 g/mo & CYC $0.6 \mathrm{~g} / \mathrm{mo}$ & CYC $0.6 \mathrm{~g} / \mathrm{mo}$ \\
\hline & HCQ $400 \mathrm{mg} / \mathrm{d}$ & HCQ 400 mg/d & HCQ 400 mg/d & HCQ 300 mg/d & HCQ $300 \mathrm{mg} / \mathrm{d}$ \\
\hline \multirow[t]{2}{*}{37} & Pred 20 mg/d & Pred 15 mg/d & Pred 15 mg/d & Pred $15 \mathrm{mg} / \mathrm{d}$ & Pred $20 \mathrm{mg} / \mathrm{d}$, \\
\hline & LEF 20 mg/d & LEF 20 mg/d & LEF 20 mg/d & LEF 20 mg/d & LEF $20 \mathrm{mg} / \mathrm{d}$ \\
\hline \multirow[t]{3}{*}{38} & Pred 10 mg/d & Pred 10 mg/d & Pred 10 mg/d & Pred $7.5 \mathrm{mg} / \mathrm{d}$ & Pred $7.5 \mathrm{mg} / \mathrm{d}$ \\
\hline & CYC 0.8 g/mo & $\mathrm{CYC} 0.8 \mathrm{~g} / \mathrm{mo}$ & CYC $0.8 \mathrm{~g} / \mathrm{mo}$ & CYC $0.8 \mathrm{~g} / \mathrm{mo}$ & $\mathrm{CYC} 0.6 \mathrm{~g} / \mathrm{mo}$ \\
\hline & HCQ 200 mg/d & HCQ 200 mg/d & HCQ 200 mg/d & HCQ 200 mg/d & HCQ 200 mg/d \\
\hline \multirow[t]{3}{*}{39} & Pred 20 mg/d & Pred 15 mg/d & Pred 10 mg/d & Pred $10 \mathrm{mg} / \mathrm{d}$ & Pred $10 \mathrm{mg} / \mathrm{d}$ \\
\hline & CYC 0.8 g/mo & CYC 0.8 g/mo & CYC $0.8 \mathrm{~g} / \mathrm{mo}$ & CYC $0.8 \mathrm{~g} / \mathrm{mo}$ & $\mathrm{CYC} 0.8 \mathrm{~g} / \mathrm{mo}$ \\
\hline & HCQ 200 mg/d & HCQ 200 mg/d & HCQ 200 mg/d & HCQ 200 mg/d & HCQ 200 mg/d \\
\hline \multirow[t]{2}{*}{40} & Pred $10 \mathrm{mg} / \mathrm{d}$ & Pred 10 mg/d & Pred 10 mg/d & Pred $10 \mathrm{mg} / \mathrm{d}$ & Pred $5 \mathrm{mg} / \mathrm{d}$ \\
\hline & LEF $20 \mathrm{mg} / \mathrm{d}$ & LEF $20 \mathrm{mg} / \mathrm{d}$ & LEF 20 mg/d & LEF 20 mg/d & LEF $20 \mathrm{mg} / \mathrm{d}$ \\
\hline
\end{tabular}

${ }^{a}$ CSA Cyclosporine A; CYC, Cyclophosphamide; HCQ, Hydroxychloroquine; LEF, Leflunomide; MMF, Mycophenolate mofetil; MP, methylprednisone; MSCT, Mesenchymal stem cell transplantation; Pred, Prednisone; UC MSC, Umbilical cord-derived mesenchymal stem cells. Patients 1 to 26 were enrolled from the Department of Rheumatology, Affiliated Drum Tower Hospital, Nanjing University Medical School, Nanjing, China. Patients 27 to 32 were enrolled from the Department of Rheumatology, Affiliated Hospital of Jiangsu University, Zhenjiang, China. Patients 33 to 37 were enrolled from the Department of Rheumatology, Subei People's Hospital of Jiangsu Province, Yangzhou, China. Patients 38 to 40 were enrolled from the Department of Rheumatology, Jiangsu Provincial People's Hospital, Nanjing, China.

the improvements in renal function. Second, this study is not a randomized controlled trial. It lacks a group of patients who received conventional therapies, but not combined with allogeneic MSC infusion. Therefore, the current data provide evidence only that allogeneic MSCT could induce renal remission on the basis of other drugs taken by patients enrolled in this study. Third, because of the differences in patients' conditions at the time of enrollment, we cannot be sure of the uniformity and standards for quality control between the different centers or different patients. We will consider performing a multicenter randomized controlled study in China to assess the safety and efficacy of MSCT in LN patients to compare the clinical safety and efficacy of combined steroid/MSC treatment and combined steroid/traditional immunosuppressive drug therapy such as CYC. In the forthcoming trial, repeated renal biopsy will be designed to further determine whether MSCT can alleviate renal pathology in LN patients. In addition, we will try to ensure uniformity among the enrolled patients for quality control. 


\section{Conclusions}

Our multicenter clinical study illustrates the safety and efficacy of systemic administration of UC MSCs in SLE patients. Moreover, a repeated MSC infusion is feasible and necessary after 6 months to avoid disease relapse.

\section{Abbreviations}

ANA: Antinuclear antibody; anti-dsDNA: Anti-double-stranded DNA antibody; BILAG: British Isles Lupus Assessment Group; BlyS: B-lymphocyte stimulator; CsA: Cyclosporine A; CYC: Cyclophosphamide; HCQ: Hydroxychloroquine; HSCT: hematopoietic stem cell transplantation; LEF: Leflunomide; LN: Lupus nephritis; MCR: Major clinical response; MMF: Mycophenolate mofetil; MSC: Mesenchymal stem cell; MSCT: Mesenchymal stem cell transplantation; PCR: Partial clinical response; Pred: Prednisone; SLE: Systemic lupus erythematosus; SLEDAI: Systemic Lupus Erythematosus Disease Activity Index.

\section{Competing interests}

The authors declare that they have no competing interests.

\section{Authors' contributions}

DW, was responsible for the study conception and design, data collection and analysis and manuscript writing. JL, YZ, MZ were responsible for the study design, data collection and analysis and manuscript revision. JC was responsible for data analysis and manuscript writing. $\mathrm{XL}$ was responsible for the study conception and design, data collection and manuscript drafting. $\mathrm{XH}$ was responsible for the study conception and design and critical revision of the manuscript. SJ was responsible for the study conception and design, data collection and manuscript drafting. SS was responsible for the study conception and design and critical revision of the manuscript. LS was responsible for the study conception and design, data collection and analysis, manuscript writing and final approval of the manuscript. All authors read and approved the final manuscript.

\section{Acknowledgements}

We want to thank Dr Zhaoxia Luo, Dr Zhaojing Wang and Dr Xiaoguang Zhang from the Stem Cell Center of Jiangsu Province for providing umbilical cord mesenchymal stem cells and collection of clinical data. The study was supported by the Major International (Regional) Joint Research Project (81120108021), the National Natural Science Foundation of China (81273304) and the Jiangsu Province Kejiao Xingwei Program.

\section{Author details}

'Department of Rheumatology and Immunology, the Affiliated Drum Tower Hospital of Nanjing University Medical School, 321 Zhongshan Road, Nanjing, Jiangsu 210008, China. ${ }^{2}$ Department of Rheumatology, the Affiliated Hospital of Jiangsu University, 438 Jiefang Road, Zhenjiang 212001, China. ${ }^{3}$ Department of Rheumatology, Subei People's Hospital of Jiangsu Province, 98 Nantong West Road, 225001 Yangzhou, China. ${ }^{4}$ Department of Rheumatology, Jiangsu Provincial People's Hospital, 300 Guangzhou Road, 210029 Nanjing, China. ${ }^{5}$ Stem Cell Center of Jiangsu Province, Taizhou, China. ${ }^{6}$ Center for Craniofacial Molecular Biology, School of Dentistry, University of Southern California Los Angeles, Los Angeles, CA, USA.

Received: 7 June 2013 Accepted: 26 February 2014

Published: 25 March 2014

\section{References}

1. Petri M: Systemic lupus erythematosus: 2006 update. J Clin Rheumatol 2006, 12:37-40.

2. Hoffman RW: T cells in the pathogenesis of systemic lupus erythematosus. Clin Immunol 2004, 113:4-13.

3. Sanz I: Connective tissue diseases: targeting B cells in SLE: good news at last! Nat Rev Rheumatol 2011, 7:255-256.

4. Liu Z, Davidson A: Taming lupus-a new understanding of pathogenesis is leading to clinical advances. Nat Med 2012, 18:871-882.

5. Rahman A, Isenberg DA: Systemic lupus erythematosus. N Engl J Med 2008, 358:929-939.

6. Karim MY, Pisoni CN, Khamashta MA: Update on immunotherapy for systemic lupus erythematosus - what's hot and what's not. Rheumatology (Oxford) 2009, 48:332-341.
7. Merrill JT, Ginzler EM, Wallace DJ, MCKay JD, Lisse JR, Aranow C, Wellborne FR, Burnette M, Condemi J, Zhong ZJ, Pineda L, Klein J, Freimuth WW, LBSL02/99 Study Group: Long-term safety profile of belimumab plus standard therapy in patients with systemic lupus erythematosus. Arthritis Rheum 2012, 64:3364-3373.

8. Manzi S, Sánchez-Guerrero J, Merrill JT, Furie R, Gladman D, Navarra SV, Ginzler EM, D'Cruz DP, Doria A, Cooper S, Zhong ZJ, Hough D, Freimuth W, Petri MA, BLISS-52 and BLISS-76 Study Groups: Effects of belimumab, a B lymphocyte stimulator-specific inhibitor, on disease activity across multiple organ domains in patients with systemic lupus erythematosus: combined results from two phase III trials. Ann Rheum Dis 2012, 71:1833-1838.

9. Alexander T, Thiel A, Rosen O, Massenkeil G, Sattler A, Kohler S, Mei H, Radtke H, Gromnica-lhle E, Burmester GR, Arnold R, Radbruch A, Hiepe F: Depletion of autoreactive immunologic memory followed by autologous hematopoietic stem cell transplantation in patients with refractory SLE induces long-term remission through de novo generation of a juvenile and tolerant immune system. Blood 2009, 113:214-223.

10. Prockop DJ: Marrow stromal cells as stem cells for nonhematopoietic tissues. Science 1997, 276:71-74.

11. Loh Y, Oyama Y, Statkute L, Quigley K, Yaung K, Gonda E, Barr W, Jovanovic B, Craig R, Stefoski D, Cohen B, Burt RK: Development of a secondary autoimmune disorder after hematopoietic stem cell transplantation for autoimmune diseases: role of conditioning regimen used. Blood 2007, 109:2643-2648.

12. Conget PA, Minguell Jj: Phenotypical and functional properties of human bone marrow mesenchymal progenitor cells. J Cell Physiol 1999, 181:67-73.

13. Sanchez-Ramos J, Song S, Cardozo-Peleza F, Hazzi C, Stedeford T, Willing A, Freeman TB, Saporta S, Janssen W, Patel N, Cooper DR, Sanberg PR: Adult bone marrow stromal cells differentiate into neural cells in vitro. Exp Neurol 2000, 164:247-256.

14. Saulnier N, Lattanzi W, Puglisi MA, Pani G, Barba M, Piscaglia AC, Giachelia M, Alfieri S, Neri G, Gasbarrini G, Gasbarrini A: Mesenchymal stromal cells multipotency and plasticity: induction toward the hepatic lineage. Eur Rev Med Pharmacol Sci 2009, 13:71-78.

15. Fan L, Lin C, Zhuo S, Chen L, Liu N, Luo Y, Fang J, Huang Z, Lin Y, Chen J: Transplantation with survivin-engineered mesenchymal stem cells results in better prognosis in a rat model of myocardial infarction. Eur $\mathrm{J}$ Heart Fail 2009, 11:1023-1030.

16. Keating A: Mesenchymal stromal cells: new directions. Cell Stem Cell 2012, 10:709-716.

17. Le Blanc K, Mougiakakos D: Multipotent mesenchymal stromal cells and the innate immune system. Nat Rev Immunol 2012, 12:383-396.

18. English K, French A, Wood KJ: Mesenchymal stromal cells: facilitators of successful transplantation? Cell Stem Cell 2010, 7:431-442.

19. Sun L, Wang D, Liang J, Zhang H, Feng $X$, Wang H, Hua B, Liu B, Ye S, Hu X, Xu W, Zeng X, Hou Y, Gilkeson GS, Silver RM, Lu L, Shi S: Umbilical cord mesenchymal stem cell transplantation in severe and refractory systemic lupus erythematosus. Arthritis Rheum 2010, 62:2467-2475.

20. Liang J, Zhang H, Hua B, Wang H, Lu L, Shi S, Hou Y, Zeng X, Gilkeson GS, Sun L: Allogeneic mesenchymal stem cells transplantation in refractory systemic lupus erythematosus: a pilot clinical study. Ann Rheum Dis 2010, 69:1423-1429. A published erratum appears in Ann Rheum Dis.

21. Wang D, Zhang H, Liang J, Li X, Feng X, Wang H, Hua B, Liu B, Lu L, Gilkeson GS, Silver RM, Chen W, Shi S, Sun L: Allogeneic mesenchymal stem cell transplantation in severe and refractory systemic lupus erythematosus: 4 years of experience. Cell Transplant 2013, 22:2267-2277.

22. Boumpas DT, Austin HA 3rd, Vaughn EM, Klippel JH, Steinberg AD, Yarboro CH, Balow JE: Controlled trial of pulse methylprednisolone versus two regimens of pulse cyclophosphamide in severe lupus nephritis. Lancet 1992, 340:741-745.

23. Illei GG, Austin HA, Crane M, Collins L, Gourley MF, Yarboro CH, Vaughan EM, Kuroiwa T, Danning CL, Steinberg AD, Klippel JH, Balow JE, Boumpas DT: Combination therapy with pulse cyclophosphamide plus pulse methylprednisolone improves long-term renal outcome without adding toxicity in patients with lupus nephritis. Ann Intern Med 2001, 135:248-257.

24. Chan TM, Li FK, Tang CS, Wong RW, Fang GX, Ji YL, Lau CS, Wong AK, Tong MK, Chan KW, Lai KN, Hong Kong-Guangzhou Nephrology Study Group: 
Efficacy of mycophenolate mofetil in patients with diffuse proliferative lupus nephritis. N Engl J Med 2000, 343:1156-1162.

25. Weening JJ, D'Agati VD, Schwartz MM, Seshan SV, Alpers CE, Appel GB, Balow JE, Bruijn JA, Cook T, Ferrario F, Fogo AB, Ginzler EM, Hebert L, Hill G, Hill P, Jennette JC, Kong NC, Lesavre P, Lockshin M, Looi LM, Makino H, Moura LA, Nagata M: The classification of glomerulonephritis in systemic lupus erythematosus revisited. J Am Soc Nephrol 2004, 15:241-250. A published erratum appears in J Am Soc Nephrol 2004, 15:835-836.

26. Merrill JT, Neuwelt CM, Wallace DJ, Shanahan JC, Latinis KM, Oates JC, Utset TO, Gordon C, Isenberg DA, Hsieh HJ, Zhang D, Brunetta PG: Efficacy and safety of rituximab in moderately-to-severely active systemic lupus erythematosus: the randomized, double-blind, phase II/III systemic lupus erythematosus evaluation of rituximab trial. Arthritis Rheum 2010, 62:222-233.

27. Symmons DP, Coppock JS, Bacon PA, Bresnihan B, Isenberg DA, Maddison P, McHugh N, Snaith ML, Zoma AS, British Isles Lupus Assessment Group (BILAG): Development and assessment of a computerized index of clinical disease activity in systemic lupus erythematosus. Q J Med 1988, 69:927-937.

28. Hay EM, Bacon PA, Gordon C, Isenberg DA, Maddison P, Snaith ML, Symmons DP, Viner N, Zoma A: The BILAG index: a reliable and valid instrument for measuring clinical disease activity in systemic lupus erythematosus. Q J Med 1993, 86:447-458.

29. Le Blanc K, Frassoni F, Ball L, Locatelli F, Roelofs H, Lewis I, Lanino E, Sundberg B, Bernardo ME, Remberger M, Dini G, Egeler RM, Bacigalupo A, Fibbe W, Ringdén O, Developmental Committee of the European Group for Blood and Marrow Transplantation: Mesenchymal stem cells for treatment of steroid-resistant, severe, acute graft-versus-host disease: a phase II study. Lancet 2008, 371:1579-1586.

30. Ball LM, Bernardo ME, Roelofs H, Lankester A, Cometa A, Egeler RM, Locatelli $F_{\text {, }}$ Fibbe WE: Cotransplantation of ex vivo-expanded mesenchymal stem cells accelerates lymphocyte recovery and may reduce the risk of graft failure in haploidentical hematopoietic stem-cell transplantation. Blood 2007, 110:2764-2767.

31. Hare JM, Fishman JE, Gerstenblith G, DiFede Velazquez DL, Zambrano JP, Suncion VY, Tracy M, Ghersin E, Johnston PV, Brinker JA, Breton E, Davis-Sproul J, Schulman IH, Byrnes J, Mendizabal AM, Lowery MH, Rouy D, Altman P, Wong Po Foo C, Ruiz P, Amador A, Da Silva J, McNiece IK, Heldman AW, George R, Lardo A: Comparison of allogeneic vs autologous bone marrow-derived mesenchymal stem cells delivered by transendocardial injection in patients with ischemic cardiomyopathy: the POSEIDON randomized trial. JAMA 2012, 308:2369-2379.

32. Song H, Song BW, Cha MJ, Choi IG, Hwang KC: Modification of mesenchymal stem cells for cardiac regeneration. Expert Opin Biol Ther 2010, 10:309-319.

33. Akiyama K, Chen C, Wang D, Xu X, Qu C, Yamaza T, Cai T, Chen W, Sun L, Shi S: Mesenchymal stem cell-induced immunoregulation involves Fasligand/Fas-mediated T cell apoptosis. Cell Stem Cell 2012, 10:544-555.

34. Liang J, Zhang H, Wang D, Feng X, Wang H, Hua B, Liu B, Sun L: Allogeneic mesenchymal stem cell transplantation in seven patients with refractory inflammatory bowel disease. Gut 2012, 61:468-469.

35. Ciccocioppo R, Bernardo ME, Sgarella A, Maccario R, Avanzini MA, Ubezio C, Minelli A, Alvisi C, Vanoli A, Calliada F, Dionigi P, Perotti C, Locatelli F, Corazza GR: Autologous bone marrow-derived mesenchymal stromal cells in the treatment of fistulising Crohn's disease. Gut 2011, 60:788-798.

36. Wang D, Zhang H, Cao M, Tang Y, Liang J, Feng X, Wang H, Hua B, Liu B, Sun L: Efficacy of allogeneic mesenchymal stem cell transplantation in patients with drug-resistant polymyositis and dermatomyositis. Ann Rheum Dis 2011, 70:1285-1288,

37. Liang J, Li X, Zhang H, Wang D, Feng X, Wang H, Hua B, Liu B, Sun L: Allogeneic mesenchymal stem cells transplantation in patients with refractory RA. Clin Rheumatol 2012, 31:157-161.

38. Xu J, Wang D, Liu D, Fan Z, Zhang H, Liu O, Ding G, Gao R, Zhang C, Ding Y, Bromberg JS, Chen W, Sun L, Wang S: Allogeneic mesenchymal stem cell treatment alleviates experimental and clinical Sjögren syndrome. Blood 2012, 120:3142-3151.

39. Ezquer FE, Ezquer ME, Parrau DB, Carpio D, Yañez AJ, Conget PA: Systemic administration of multipotent mesenchymal stromal cells reverts hyperglycemia and prevents nephropathy in type 1 diabetic mice. Biol Blood Marrow Transplant 2008, 14:631-640.
40. Jiang R, Han Z, Zhuo G, Qu X, Li X, Wang X, Shao Y, Yang S, Han ZC: Transplantation of placenta-derived mesenchymal stem cells in type 2 diabetes: a pilot study. Front Med 2011, 5:94-100.

41. Wang D, Akiyama K, Zhang H, Yamaza T, Li X, Feng X, Wang H, Hua B, Liu B, $X u H$, Chen W, Shi S, Sun L: Double allogeneic mesenchymal stem cells transplantations could not enhance therapeutic effect compared with single transplantation in systemic lupus erythematosus. Clin Dev Immunol 2012, 2012:273291

42. Tang Y, Xie H, Chen J, Geng L, Chen H, Li X, Hou Y, Lu L, Shi S, Zeng X, Sun $\mathrm{L}$ : Activated NF-KB in bone marrow mesenchymal stem cells from systemic lupus erythematosus patients inhibits osteogenic differentiation through downregulating Smad signaling. Stem Cells Dev 2013, 22:668-678.

43. Li X, Liu L, Meng D, Wang D, Zhang J, Shi D, Liu H, Xu H, Lu L, Sun L: Enhanced apoptosis and senescence of bone-marrow-derived mesenchymal stem cells in patients with systemic lupus erythematosus. Stem Cells Dev 2012, 21:2387-2394.

44. Kastrinaki MC, Sidiropoulos $P$, Roche $S$, Ringe J, Lehmann S, Kritikos H, Vlahava VM, Delorme B, Eliopoulos GD, Jorgensen C, Charbord P, Häupl T, Boumpas DT, Papadaki HA: Functional, molecular and proteomic characterisation of bone marrow mesenchymal stem cells in rheumatoid arthritis. Ann Rheum Dis 2008, 67:741-749.

45. Guiducci S, Manetti M, Romano E, Mazzanti B, Ceccarelli C, Dal Pozzo S, Milia AF, Bellando-Randone S, Fiori G, Conforti ML, Saccardi R, Ibba-Manneschi L, Matucci-Cerinic M: Bone marrow-derived mesenchymal stem cells from early diffuse systemic sclerosis exhibit a paracrine machinery and stimulate angiogenesis in vitro. Ann Rheum Dis 2011, 70:2011-2021.

46. Subramanian A, Shu-Uin G, Kae-Siang N, Gauthaman K, Biswas A, Choolani M, Bongso A, Chui-Yee F: Human umbilical cord Wharton's jelly mesenchymal stem cells do not transform to tumor-associated fibroblasts in the presence of breast and ovarian cancer cells unlike bone marrow mesenchymal stem cells. J Cell Biochem 2012, 113:1886-1895.

47. Secco M, Moreira YB, Zucconi E, Vieira NM, Jazedje T, Muotri AR, Okamoto OK, Verjovski-Almeida S, Zatz M: Gene expression profile of mesenchymal stem cells from paired umbilical cord units: cord is different from blood. Stem Cell Rev 2009, 5:387-401.

48. Kim MJ, Shin KS, Jeon JH, Lee DR, Shim SH, Kim JK, Cha DH, Yoon TK, Kim GJ: Human chorionic-plate-derived mesenchymal stem cells and Wharton's jelly-derived mesenchymal stem cells: a comparative analysis of their potential as placenta-derived stem cells. Cell Tissue Res 2011, 346:53-64.

\section{doi:10.1186/ar4520}

Cite this article as: Wang et al:: Umbilical cord mesenchymal stem cell transplantation in active and refractory systemic lupus erythematosus: a multicenter clinical study. Arthritis Research \& Therapy 2014 16:R79.

\section{Submit your next manuscript to BioMed Central and take full advantage of:}

- Convenient online submission

- Thorough peer review

- No space constraints or color figure charges

- Immediate publication on acceptance

- Inclusion in PubMed, CAS, Scopus and Google Scholar

- Research which is freely available for redistribution 